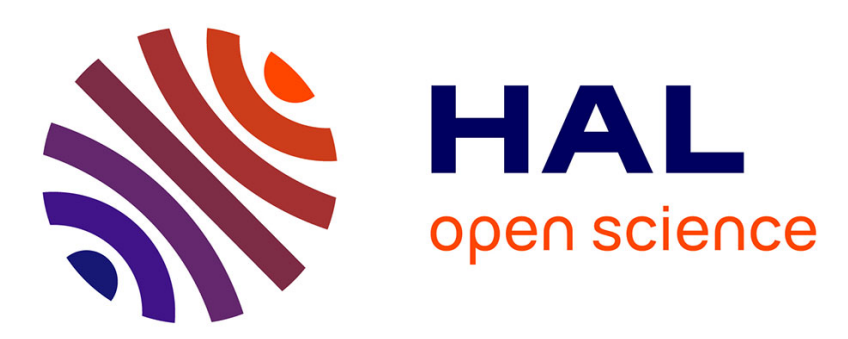

\title{
A cohesive zone model which is energetically equivalent to a gradient-enhanced coupled damage-plasticity model
}

Fabien Cazes, Anita Simatos, Michel Coret, Alain Combescure

\section{To cite this version:}

Fabien Cazes, Anita Simatos, Michel Coret, Alain Combescure. A cohesive zone model which is energetically equivalent to a gradient-enhanced coupled damage-plasticity model. European Journal of Mechanics - A/Solids, 2010, 29 (6), pp.976. 10.1016/j.euromechsol.2009.11.003 . hal-00687358

\section{HAL Id: hal-00687358 https://hal.science/hal-00687358}

Submitted on 13 Apr 2012

HAL is a multi-disciplinary open access archive for the deposit and dissemination of scientific research documents, whether they are published or not. The documents may come from teaching and research institutions in France or abroad, or from public or private research centers.
L'archive ouverte pluridisciplinaire $\mathbf{H A L}$, est destinée au dépôt et à la diffusion de documents scientifiques de niveau recherche, publiés ou non, émanant des établissements d'enseignement et de recherche français ou étrangers, des laboratoires publics ou privés. 


\section{Accepted Manuscript}

Title: A cohesive zone model which is energetically equivalent to a gradient-enhanced coupled damage-plasticity model

Authors: Fabien Cazes, Anita Simatos, Michel Coret, Alain Combescure

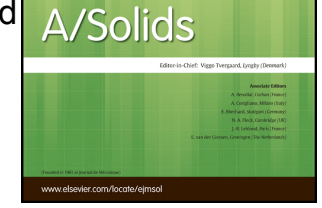

PII: S0997-7538(09)00133-8

DOI: $\quad$ 10.1016/j.euromechsol.2009.11.003

Reference: EJMSOL 2568

To appear in: European Journal of Mechanics / A Solids

Received Date: 10 June 2009

Revised Date: 4 November 2009

Accepted Date: 7 November 2009

Please cite this article as: Cazes, F., Simatos, A., Coret, M., Combescure, A. A cohesive zone model which is energetically equivalent to a gradient-enhanced coupled damage-plasticity model, European Journal of Mechanics / A Solids (2009), doi: 10.1016/j.euromechsol.2009.11.003

This is a PDF file of an unedited manuscript that has been accepted for publication. As a service to our customers we are providing this early version of the manuscript. The manuscript will undergo copyediting, typesetting, and review of the resulting proof before it is published in its final form. Please note that during the production process errors may be discovered which could affect the content, and all legal disclaimers that apply to the journal pertain. 


\title{
A cohesive zone model which is energetically equivalent to a gradient-enhanced coupled damage-plasticity model
}

\author{
Fabien Cazes ${ }^{*, a}$, Anita Simatos ${ }^{\mathrm{a}, \mathrm{b}}$, Michel Coret $^{\mathrm{a}}$, Alain Combescure ${ }^{\mathrm{a}}$ \\ ${ }^{a}$ Université de Lyon, CNRS, INSA-Lyon, LaMCoS UMR5259, F-69621, France \\ ${ }^{b}$ DM2S/SEMT/LISN, CEA Saclay, 91191 Gif-sur-Yvette, France
}

\begin{abstract}
Modeling the fracture of a material can take two different approaches. A first solution consists in using models which preserve a continuous description of the material throughout the fracture process. These models are often regularized in order to deal with the softening part of the material's behavior properly. Another solution consists in introducing discontinuity surfaces into the structure along with the possibility of taking into account cohesive forces between the two sides of the discontinuity. Many works have been devoted to the establishment of a relation between these two families of models. The present work is based on the equivalent crack concept, which states that a localized damage zone can be replaced by a crack as long as the energy dissipated by the structure is preserved when switching models. In a first paper, we introduced a method of construction of a cohesive law based on an elastic-damageable reference model. For a given test case, the cohesive model was built incrementally from the known solution given by the continuous reference model. There was no prerequisite assumption on the form of the cohesive law. In that work, the presence of plastic strains in the structure had not been taken into account, which limited the range of applicability of the method to elastic-damageable models. The objective of this paper is to eliminate this limitation by extending the method to the more general class of elastic-plastic damageable models.
\end{abstract}

Key words: equivalent crack concept, cohesive law, enhanced continuum, damage, plasticity

\section{Introduction}

The modeling of the hardening part of a material's traction curve is well-known. Depending on the material, one can assume that the internal transformations lead to the occurrence of plastic strains (elastic-plastic models) or to a drop in the material's stiffness (damage models). The actual behavior of materials is often closer to that of elasticplastic damageable models combining damage and plasticity. All these models fall within a thermodynamic framework defined in Lemaitre and Chaboche (1988). A study of wave propagation in the materials reveals that if the slope of the material's traction curve tends to zero the dissipative phenomena concentrate in a zone of zero thickness (Bažant and Belytschko, 1985). This shows that classical damage and plasticity models are incapable of modeling the behavior of a material up to rupture realistically. Furthermore, the observation of fracture shows that microcracks or cavities occur progressively within the material, which makes the definition of stresses and strains less rigorous. This leads to the question of whether it is relevant to use a stress-strain curve when softening begins to occur. This question can have several answers, which explains the

\footnotetext{
* Corresponding author

Email address: fabien.cazes@insa-lyon.fr (Fabien Cazes)
}

large number of models available to deal with a material's fracture.

First, some models retain a continuous description of the material throughout the fracture process. In this case, the use of a localization-limiting scheme enables one to prescribe a lower limit to the width of the damage zone. This family includes, among others, nonlocal models (PijaudierCabot and Bažant, 1987), of which second-gradient models (Aifantis, 1984) can be considered to be a subclass (Peerlings et al., 2001), and delay-effect models (Ladevèze, 1992). One can also consider that fracture is due to the initiation and propagation of discontinuity surfaces within the structure. In the first such model, introduced by Griffith (1920), the cracks' faces are assumed to be free from external loads when writing the equilibrium equations, while an energy criterion states whether a crack propagates or not. Cohesive models were introduced later by Barenblatt (1962) and Dugdale (1960) in order to achieve more realistic stress and strain fields at the cracks' tips. In these models, a law expressing the stress vector as a function of the displacement jump at a point of the discontinuity is applied. These models have been successfully extended to quasi-brittle materials (Hillerborg et al., 1976), in which case they give a relatively coarse representation of the physical phenomena involved in the fracture process.

The choice of one model over another is often based on 
practical criteria, such as the ease with which the material parameters can be identified or the numerical implementation carried out. Continuous models seem to provide a more precise description of the material's behavior because the dissipative phenomena can be spread over a volume, whereas in cohesive models they are assumed to be condensed onto a surface. The numerical implementation of regularized continuous models can be time consuming because of the regularisation step (non-local model) or the inversion of non-symetric matrices (as often for gradientmodels). The results are satisfactory only if the mesh is sufficiently refined so that several elements run across the damage zone, which may require the use of remeshing techniques. (See, e.g., Rodríguez-Ferran and Huerta (2000); Patzák and Jirásek (2004)). On the other hand, numerical implementation of discontinous models is efficient thanks to the numerous research efforts which have been devoted to them. For exemple, the X-FEM method allow great flexibility in the crack's path and can be used with rather coarse meshes. They were first applied to Griffith's theory (Belytschko and Black, 1999; Moës et al., 1999) and then extended to cohesive zone models (Wells and Sluys, 2001; Moës and Belytschko, 2002; Mariani and Perego, 2003).

Sevel papers have been devoted to the use of both a continuous and a discontinuous model in calculations. A first solution is to introduce a discontinuity into the structure wherever the damage is equal to 1 (de Borst and Abellan, 2002; Simone et al., 2003; Mediavilla et al., 2006). This is particularly interessing for non-local models because it prevents the nonlocal coupling of points located on both sides of the discontinuity. The strong discontinuity formalism (Simo et al., 1993; Oliver, 1996) allows continuous models and discontinuous models similar to cohesive zones to be used alongside one another in order to treat the material's behavior in its hardening and softening parts separately. The weak discontinuity concept can also be used to perform a progressive transition from a continuous description to a discontinuous description if the width of the weak discontinuity varies and tends toward zero at the end of the fracture process. This method was used in Oliver et al. (2002) with a numerical implementation of the embedded-discontinuity type, and in Benvenuti et al. (2008) in the framework of the X-FEM. With those methodes continuous and discontinuous models are juxtaposed next to one another.

There is another family of methods in which the continuous and discontinuous models are superimposed, the discontinuous model being built from the continuous model in order to represent the same phenomena. In this family, a first model substitution method was introduced specifically for nonlocal models of the integral type in Planas et al. (1993). The idea was to use the regularizing properties of nonlocal integration to go from a discontinuous field to a continuous field. A variation of this method enables one to determine, a posteriori, the opening of a crack corresponding to a nonlocal damage state (Legrain et al., 2007; Dufour et al., 2008). Another means of obtaining a cracking model which is equivalent to a damage model consists in requiring the conservation of the dissipated energy during the model substitution. This idea inspired the equivalent crack concept, introduced in Mazars (1984) and Mazars and Pijaudier-Cabot (1996), which can be used to calculate a Griffith model equivalent to a reference damage model. In Bažant and Oh (1983), a dissipated energy equivalence was also used to calculate the parameters of a crack-band model, based on a given cohesive model, as functions of the desired bandwidth. In this case, the reverse process is applied as it is the continuous model which is calculated from the discontinuous model.

The equivalent crack concept was used to build cohesive models from damage models under certain assumptions concerning the form of the cohesive law. In order to do that, one would use the property that the area under the cohesive law is equal to the dissipated energy per unit area when the faces of the cracks are completely disconnected. The discontinuity can be introduced into the damaged material when some localization criterion is verified (Areias and Belytschko, 2005) or when the mesh is no longer refined enough to describe the damage profile appropriately (Comi et al., 2002, 2007). In this case, the cohesive zone receives the amount of energy which remains to be dissipated by the continuous material until its fracture. One should note that these works combine the juxtaposition and superposition of continuous and discontinuous models.

The equivalent crack concept was used in a previous paper (Cazes et al., 2009) to build a cohesive law from a given, regularized elastic-damageable model. A study of the domains of validity of the models being considered leads to the definition of an equivalent problem in which the nonlocal regularization of the reference problem is replaced by the occurrence of a cohesive zone in the structure (Figure 1).

\begin{tabular}{|c|c|c|}
\hline & Diffuse damage & Localized damage \\
\hline $\begin{array}{l}\text { Reference } \\
\text { model }\end{array}$ & Continuous damage & + Regularization \\
\hline $\begin{array}{l}\text { Equivalent } \\
\text { model }\end{array}$ & Continuous damage & Cohesive zone \\
\hline
\end{tabular}

Figure 1: The reference model and the equivalent model

Then, a model substitution criterion is defined in order for the energy dissipated in the softening part of the material's behavior to be transferred to the cohesive zone of the equivalent model as represented in Figure 2. 


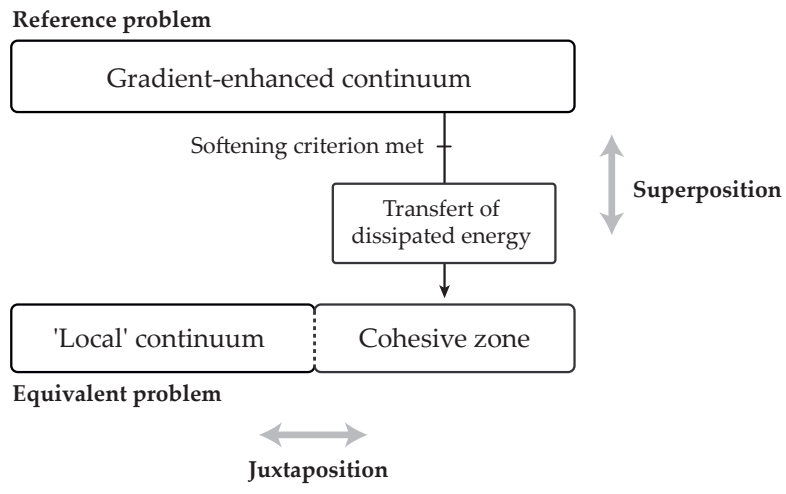

Figure 2: The proposed model substitution method

The first step consists in determining a solution of the regularized reference problem. This resolution involves the calculation of the increments of dissipated energy between two time steps. At the same time, one carries out a calculation with the equivalent model. The path of the equivalent crack is assumed to be known beforehand but the cohesive law is an unknown of the problem. The solution at time $t+d t$ is obtained from the solution at time $t$ and the prescribed increment of dissipated energy between $t$ and $t+d t$. Before this calculation, the cohesive model is completely unknown and no assumption is made about the form of the cohesive law. Under certain assumptions, it is possible to show that all the terms involved in the energy balance of the structure are preserved when switching models.

The objective of this paper is to extend this method to models combining plasticity and damage. First, we will modify the thermodynamic description of the discontinuity in order to account for the presence of a plastic displacement jump between the two faces of the crack. Next, the model substitution criterion based on the dissipated energy will be modified in order to enforce the energies dissipated through plasticity and damage independently. Then, this new model substitution criterion along with some additional assumptions will be used to restore the conservation of the energy balance terms of the reference model. In the last part, the method will be applied to the construction of the cohesive law starting from a onedimensional implicit second-gradient model.

\section{Study of the thermodynamics of the cohesive zone}

Let us consider a continuous domain $\Omega$ bounded by $\Gamma$ and traversed by a discontinuity surface $\Gamma_{s}$. The orientation of the crack is given by a unit vector $\underline{n}$ perpendicular to the discontinuity surface (Figure 3 ). This vector enables one to identify the upper and lower faces, denoted $\Gamma_{s}^{+}$and $\Gamma_{s}^{-}$respectively. The displacement jump $\llbracket \underline{u} \rrbracket$ is equal to the difference between the displacements $\underline{u}^{+}$and $\underline{u}^{-}$observed over $\Gamma_{s}^{+}$and $\Gamma_{s}^{-}$:

$$
\llbracket \underline{u} \rrbracket=\underline{u}^{+}-\underline{u}^{-}, \quad \text { over } \Gamma_{s} .
$$

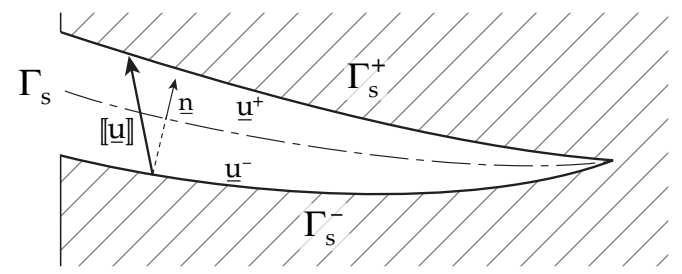

Figure 3: Orientation of the cracks and notations

With a stress vector $\underline{\sigma}^{s}$ defined over $\Gamma_{s}$, the equilibrium equations at the discontinuity are

$\underline{\sigma}^{s}=\underline{\underline{\sigma}}^{+} \underline{n}=\underline{\underline{\sigma}}^{-} \underline{n}, \quad$ over $\Gamma_{s}$.

The heat flow jump is defined by

$\llbracket \underline{q} \rrbracket=\left(\underline{q}^{+}-\underline{q}^{-}\right) \underline{n}, \quad \quad$ over $\Gamma_{s}$.

A thermodynamic framework similar to that defined for continuous media (Lemaitre and Chaboche, 1988) is used for the discontinuity. From Gurtin (1979), the local expressions of the first and second principles of thermodynamics are

$$
\begin{aligned}
& \frac{d e_{s}}{d t}=\frac{\sigma^{s}}{d \llbracket \underline{u} \rrbracket} \frac{\llbracket q \underline{ } \rrbracket}{d t}, \\
& \frac{d s_{s}}{d t}=\frac{\llbracket \underline{q} \rrbracket \underline{n}}{T}+\frac{d\left(s_{i}\right)_{s}}{d t} .
\end{aligned}
$$

where $t$ is the time, $T$ the temperature, and $e_{s}, s_{s},\left(s_{i}\right)_{s}$ are the surface densities of internal energy, entropy and internal entropy respectively. By definition, the surface energy dissipated by the cohesive zone is

$d \phi_{s}=T d\left(s_{i}\right)_{s}$

Using 4, 5 and the following definition of the free surface energy:

$\psi_{s}=e_{s}-T s_{s}$,

one gets the expression of a time increment of dissipated energy:

$d \phi_{s}=\underline{\sigma}^{s} d \llbracket \underline{u} \rrbracket-d \psi_{s}-s_{s} d T$.

Costanzo and Allen (1995) define the cohesive zone in the context of standard generalized materials and introduce irreversible cohesive stresses. In this work, the use of a plastic displacement jump $\llbracket u \rrbracket^{p}$ was preferred because of the analogy which can be made with the plastic strain $\underline{\underline{\varepsilon}}^{p}$. Thus, the displacement jump $\llbracket u \rrbracket$ is divided into

$\llbracket \underline{u} \rrbracket=\llbracket \underline{u} \rrbracket^{e}+\llbracket \underline{u} \rrbracket^{p}$.

This constitutes the first difference from Cazes et al. (2009), in which the plastic displacement was assumed to be zero in order to be consistent with the elasticdamageable reference model. Let $v^{k}$ denote the internal 
variables defined at the discontinuity. Starting from the general form of the free surface energy

$\psi_{s}=\psi_{s}\left(\llbracket \underline{u} \rrbracket^{e}, T, v^{k}\right)$,

classical energy reasoning (such as Lemaitre and Chaboche (1988)) enables one to show that

$\underline{\sigma}^{s}=\frac{\partial \psi_{s}}{\partial \llbracket \underline{u} \rrbracket^{e}}, \quad$ and $\quad s_{s}=-\frac{\partial \psi_{s}}{\partial T}$.

\section{The models being considered}

\subsection{The continuous reference model}

The reference model we use, which is similar to a model presented in de Borst et al. (1999), is a second-gradient implicit model in which damage is coupled with plasticity. The specific free potential energy $\psi$ is assumed to be of the form

$\rho \psi=\frac{1}{2}(1-D) \underline{\underline{\varepsilon}}^{e}: \mathbf{K}: \underline{\underline{\varepsilon}}^{e}$,

where $\mathrm{D}$ is the scalar damage variable, $\underline{\underline{\varepsilon}}^{e}$ the elastic part of the linearized strain tensor and $\mathbf{K}$ the Hooke's tensor of the virgin material. With this form of the potential, the temperature has no effect on the material's behavior. No free energy is stored in the material's microstructure because if the elastic strain becomes zero at a point, so does the specific free energy. The form of the potential leads to the following expression of the stress $\underline{\underline{\sigma}}$ :

$\underline{\underline{\sigma}}=\rho \frac{\partial \psi}{\partial \underline{\underline{\varepsilon}}^{e}}=(1-D) \mathbf{K}: \underline{\underline{\varepsilon}}^{e}$.

The evolution of plasticity is governed by the effective stress, defined by

$\underline{\underline{\tilde{\sigma}}}=\mathbf{K}: \underline{\underline{\varepsilon}}^{e}$,

which corresponds to the stress that would exist in the material with the same strain state in the absence of damage. The only internal variable of the model is the accumulated plastic strain $p$ defined incrementally by

$d p=\sqrt{\frac{2}{3}}\left\|d \underline{\underline{\varepsilon}}^{p}\right\|$

where $\|$.$\| is a norm such that \|\underline{\underline{a}}\|=\sqrt{\underline{\underline{a}}: \underline{\underline{a}}}$. The elastic domain is described by the threshold function $f$ such that any stress state verifies

$f(\underline{\underline{\tilde{\sigma}}}, p) \leq 0$.

The plasticity evolution laws are defined such that the model shows hardening behavior in the absence of damage. The damage variable is expressed as a function of the memory variable $\kappa$ calculated from a regularized variable $\bar{z}$ such that

$\bar{z}-c \nabla^{2} \bar{z}=z$. where $\nabla^{2}$ denotes Laplace's operator, $z$ a variable to be regularized, and $c$ a characteristic quantity, homogeneous to the square of a distance, which sets the minimum size of the damage zone. The memory variable $\kappa$ is equal to the maximum value of $\bar{z}$ over the loading history:

$\kappa=\max _{t}(\bar{z})$

In order for the model to be defined completely, we still need the expressions of

- the threshold function $f$,

- the damage $D$ as a function of $\kappa$,

- the variable $z$.

An increment of dissipated volume energy is equal to

$d \phi=\underline{\underline{\sigma}}: d \underline{\underline{\varepsilon}}^{p}-Y d D$,

where $Y$ is the elastic energy recovery rate which verifies

$Y=\frac{\partial \psi}{\partial D}=-\frac{1}{2} \underline{\underline{\varepsilon}}^{e}: \mathbf{K}: \underline{\underline{\varepsilon}}^{e}$.

This incremental energy can be divided into a plastic contribution which, here, is equal to a plastic work increment, and an elastic contribution due to the damage:

$d \phi=d \phi^{p}+d \phi^{e}$

with

$d \phi^{p}=\underline{\underline{\sigma}}: d \underline{\underline{\varepsilon}}^{p} \quad$ and $\quad d \phi^{e}=-Y d D$.

Note: The dissipated energy $d \phi^{e}$ caused by the damage can also be calculated through the formula

$d \phi^{e}=\frac{1}{2}\left(\underline{\underline{\sigma}}: d \underline{\underline{\varepsilon}}^{e}-\underline{\underline{\varepsilon}}^{e}: d \underline{\underline{\sigma}}\right)$.

\subsection{The equivalent model with a cohesive zone}

For the continuous part of the equivalent model, the constitutive relations are the same as those of the reference model, the only difference being that the memory variable $\kappa$ is calculated directly from the equivalent strain $z$ without going through the regularization stage:

$\kappa=\max _{t}(z)$.

As long as one remains within the hardening part of the material's behavior, one can consider the regularized and unregularized models to be identical because the damage remains diffuse and the term $\nabla^{2} \bar{z}$ of Equation 17 is negligible.

When one enters the softening part of the material's behavior, the regularization of the equivalent strain of the reference model is replaced by the occurrence of a discontinuity in the equivalent model. The free potential energy of the discontinuous model is assumed to depend on the 
elastic part of the displacement jump $\llbracket \underline{u} \rrbracket^{e}$ and on internal variables denoted $v^{k}$ :

$\psi_{s}=\psi_{s}\left(\llbracket \underline{u} \rrbracket^{e}, v^{k}\right)$.

The threshold function $f_{s}$ is defined such that any material state satisfies

$f_{s}\left(\underline{\tilde{\sigma}}^{s}, v^{k}\right) \leq 0$

In order to be consistent with the damageable elasticplastic reference model, the stress $\underline{\sigma}^{s}$ must be a linear function of the elastic displacement jump within the elastic domain. Therefore, we assume that there exists a symmetrical operator $\underline{\underline{k}}$, which is a function of the internal variables $v^{k}$ alone, such that

$\underline{\sigma}^{s}=\underline{\underline{k}}\left(v^{k}\right) \llbracket \underline{u} \rrbracket^{e}$.

This stress value can be retrieved from Equation 11 by choosing a free surface potential energy $\psi_{s}$ of the form

$\psi_{s}=\frac{1}{2} \llbracket \underline{u} \rrbracket^{e} \underline{\underline{k}}\left(v^{k}\right) \llbracket \underline{u} \rrbracket^{e}$.

According to the reference model, no energy is stored in the material's microstructure because if $\llbracket \underline{u} \rrbracket^{e}$ is zero, then $\psi_{s}$ is also zero. Replacing $\underline{\sigma}^{s}$ in 28 by its value given in 27 , we get

$\psi_{s}=\frac{1}{2} \underline{\sigma}^{s} \llbracket \underline{u} \rrbracket^{e}$.

This expression of $\psi_{s}$ has the particularity of being independent of $v^{k}$ as in 28. Introducing this expression into 8 , we get the following expression of an increment of dissipated energy:

$d \phi_{s}=\underline{\sigma}^{s} d \llbracket \underline{u} \rrbracket^{p}+\frac{1}{2}\left(\underline{\sigma}^{s} d \llbracket \underline{u} \rrbracket^{e}-\llbracket \underline{u} \rrbracket^{e} d \underline{\sigma}^{s}\right)$.

In this expression, one can distinguish the contribution of the evolution of the plastic displacement jump $\llbracket \underline{u} \rrbracket^{p}$ and of the elastic displacement jump $\llbracket \underline{u} \rrbracket^{e}$, denoted $d \phi_{s}^{p}$ and $d \phi_{s}^{e}$ respecively:

$d \phi_{s}=d \phi_{s}^{p}+d \phi_{s}^{e}$

with,

$d \phi_{s}^{p}=\underline{\sigma}^{s} d \llbracket \underline{u} \rrbracket^{p}$,

$d \phi_{s}^{e}=\frac{1}{2}\left(\underline{\sigma}^{s} d \llbracket \underline{u} \rrbracket^{e}-\llbracket \underline{u} \rrbracket^{e} d \underline{\sigma}^{s}\right)$.

This decomposition of the increments of dissipated energy is shown in Figure 4 for a one-dimensional model.

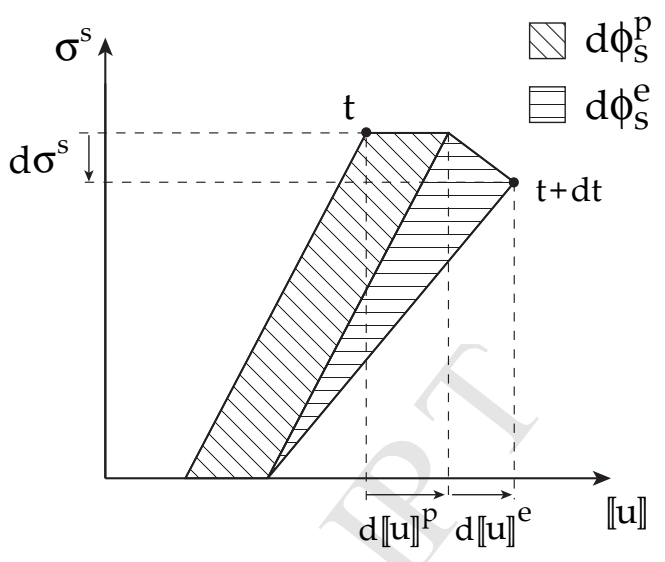

Figure 4: Increments of plastic and elastic dissipated energy

\section{The method for model substitution}

The previous two sections enabled us to define the reference model and the equivalent model in a similar thermodynamic framework, which eases the transfer of information between these two models. The models were also constructed in such a way that they verify the same assumptions:

- no energy is stored in the material's microstructure,

the behavior over the loading surface is linear.

Now, we must define criteria for the construction of the cohesive law of the equivalent model from the solution of the reference problem.

\subsection{The model substitution criterion}

Let us define the plastic and elastic dissipated energies $\Phi^{p}$ and $\Phi^{e}$ integrated over the whole domain as

$\begin{aligned} \Phi^{p} & =\int_{\Omega} \phi^{p} d V+\int_{\Gamma_{s}} \phi_{s}^{p} d S, \\ \Phi^{e} & =\int_{\Omega} \phi^{e} d V+\int_{\Gamma_{s}} \phi_{s}^{e} d S .\end{aligned}$

For the reference model, the contribution of the integrals over $\Gamma_{s}$ is zero because this model has no discontinuities. According to 21 and 31, the total dissipated energy $\Phi$ verifies

$\Phi=\Phi^{p}+\Phi^{e}$

Let $(\because)$ denote the dissipated energies of the reference model and ( $($.$) the dissipated energies of the equivalent$ model. The model substitution criterion must be defined in such a way that at any time

$\left[\begin{array}{l}d \hat{\Phi}^{p} \\ d \hat{\Phi}^{e}\end{array}\right]=\left[\begin{array}{l}d \breve{\Phi}^{p} \\ d \breve{\Phi}^{e}\end{array}\right]$

One introduces a localized damage indicator $I_{l o c}$ which is initially zero and takes the value 1 as soon as softening 
behavior occurs at a point of the structure. This can be characterized by the strict loss of positivity of the product $d \sigma^{l o c}: d \varepsilon$, where $d \sigma^{l o c}$ is the stress increment which would occur in the absence of a localization limiter. Thus, one has

$I_{l o c}=\operatorname{Arg}\left(d \underline{\underline{\sigma}}^{l o c}: d \underline{\underline{\varepsilon}}<0\right)$.

The verification of this criterion means that material stability would be lost in the absence of the localization limiter. In the case of diffuse damage, the (nonlocal) reference model and the (local) equivalent model are assumed to lead to the same result, and no energy needs to be transferred into the cohesive zone. Therefore, if $\hat{\Phi}_{s}^{e}$ and $\hat{\Phi}_{s}^{p}$ denote respectively the elastic and plastic energies dissipated over the discontinuity surface of the equivalent model, the model substitution criterion being used is

$$
\left[\begin{array}{l}
d \hat{\Phi}_{s}^{p} \\
d \hat{\Phi}_{s}^{e}
\end{array}\right]=I_{l o c}\left[\begin{array}{l}
d \breve{\Phi}^{p} \\
d \breve{\Phi}^{e}
\end{array}\right] .
$$

\subsection{Definition of a fictitious transformation and rewriting of the model substitution criterion}

In this section, we redefine the model substitution criterion using a fictitious transformation defined between the consecutive times $t$ and $t+d t$. The state of the material at time $t$ is assumed to be the same for the actual transformation and the fictitious transformation. Between $t$ and $t+d t$, the fictitious transformation is identical to the actual transformation from a mechanical standpoint, but it occurs with no change of state of the unloaded structure, i.e. with no change of the variables $\underline{\varepsilon}^{p}$ and $\llbracket \underline{u} \rrbracket^{p}$ associated with plasticity. The increments of volume and surface energy dissipated by the fictitious transformation are designated by $d \phi^{f}$ and $d \phi_{s}^{f}$. The other quantities associated with that transformation are denoted (-) and verify, for the continuous part of the model

$$
\begin{aligned}
d \underline{\underline{\varepsilon}} & =d \underline{\underline{\varepsilon}} \\
d \underline{\bar{\sigma}} & =d \underline{\underline{\sigma}} \\
d \underline{\underline{\bar{\varepsilon}}}^{p} & =\underline{\underline{0}}
\end{aligned}
$$

The increment of fictitious dissipated energy $d \phi^{f}$ can be calculated from the increments of the fictitious transformation using 21, 22 and 23:

$d \phi^{f}=\underline{\underline{\sigma}}: d \underline{\underline{\varepsilon}}^{p}+\frac{1}{2}\left(\underline{\underline{\sigma}}: d \underline{\underline{\varepsilon}}^{e}-\underline{\underline{\varepsilon}}^{e}: d \underline{\underline{\sigma}}\right)$.

Using 40, 41 and 42, one can calculate the fictitious dissipated energy from the variables of the actual transformation:

$d \phi^{f}=\frac{1}{2}\left(\underline{\underline{\sigma}}: d \underline{\underline{\varepsilon}}-\underline{\underline{\varepsilon}}^{e}: d \underline{\underline{\sigma}}\right)$.

One can bring out in this equation the expressions of $d \phi^{p}$ and $d \phi^{e}$ given by 22 and 23 . This leads to

$d \phi^{f}=\frac{1}{2} d \phi^{p}+d \phi^{e}$.
In the case where the domain includes a discontinuity (equivalent problem), one also has

$$
\begin{aligned}
d \llbracket \underline{\bar{u}} \rrbracket & =d \llbracket \underline{u} \rrbracket, \\
d \overline{\bar{\sigma}}^{s} & =d \underline{\sigma}^{s}, \\
d \llbracket \bar{u} \rrbracket^{p} & =\underline{0} .
\end{aligned}
$$

The dissipated energy $d \phi_{s}^{f}$ is calculated using 30 with the fictitious transformation increments:

$d \phi_{s}^{f}=\underline{\sigma}^{s} d \llbracket \underline{u} \rrbracket^{p}+\frac{1}{2}\left(\underline{\sigma}^{s} d \llbracket \underline{\bar{u}} \rrbracket^{e}-\llbracket \underline{u} \rrbracket^{e} d \underline{\bar{\sigma}}^{s}\right)$.

Using Equations 46, 47 and 48, one can express $d \phi_{s}^{f}$ with the variables of the actual transformation:

$d \phi_{s}^{f}=\frac{1}{2}\left(\underline{\sigma}^{s} d \llbracket \underline{u} \rrbracket-\llbracket \underline{u} \rrbracket^{e} d \underline{\sigma}^{s}\right)$.

This equation can be rewritten using the expressions of $d \phi_{s}^{p}$ and $d \phi_{s}^{e}$ given in 32 and 33:

$d \phi_{s}^{f}=\frac{1}{2} d \phi_{s}^{p}+d \phi_{s}^{e}$

Equations 45 and 51, after integration over the domain, lead to the relation between the increment of energy $d \Phi^{f}$ dissipated by the fictitious transformation and the increments of elastic and plastic dissipated energy:

$d \Phi^{f}=\frac{1}{2} d \Phi^{p}+d \Phi^{e}$.

Starting from the fictitious transformation, the model substitution criterion can be redefined as follows:

$$
\left[\begin{array}{l}
d \hat{\Phi}_{s}^{p} \\
d \hat{\Phi}_{s}^{f}
\end{array}\right]=I_{l o c}\left[\begin{array}{l}
d \breve{\Phi}^{p} \\
d \breve{\Phi}^{f}
\end{array}\right] .
$$

Using 52, one can show that this criterion, which will be used during the numerical implementation of the method and to justify the validity of the method, is equivalent to the first criterion proposed in 39 .

\section{Validity of the model substitution}

The model substitution criterion defined previously requires the equivalent model to dissipate the same amount of energy as the reference model. The ratio between plastic and elastic dissipated energy must also be preserved when switching models. The conservation of the dissipated energy is necessary for the model substitution to be valid, but in order to achieve strict energy equivalence the other terms of the energy balance must also be preserved. This energy balance, obtained in Cazes et al. (2009), can be expressed as

$\mathcal{E}=\Psi-W_{\text {ext }}+\Phi$.

where $\mathcal{E}$ is the total energy of the structure (which, by definition, is constant with time), $\Psi$ is the free energy of the 
structure and $W_{e x t}$ is the work of the external loads. In the case of elastic-damageable models, under the assumption of proportional, prescribed-displacement loading, the model substitution method preserves not only the energy dissipated by the structure, but also the free energy and the work of the external loads (Cazes et al., 2009). The purpose of this section is to obtain a similar result in the case of elastic-plastic damageable models, with the model substitution criterion defined previously and under the following assumptions:

1) the boundary conditions are prescribed loads at the boundary $\Gamma$ of the domain,

2) the loading is proportional,

3) the material does not become plastic again during unloading.

\subsection{Expressions of the free and dissipated energies}

Let the fields associated with the fully unloaded state be denoted $(.)_{r}$, where $\mathrm{r}$ means residual. This state is defined by

$\underline{\underline{\sigma}}_{r} \underline{n}=0, \quad$ over $\Gamma$.

Under Assumptions 1, 2 and 3, one can show that the free energy is

$\Psi=\Psi_{a}+\Psi_{r}$

where $\Psi_{r}$ is the remaining free energy in the unloaded state and $\Psi_{a}$ is the energy defined by

$\Psi_{a}=\frac{1}{2} \int_{\Gamma} \underline{F}\left(\underline{u}-\underline{u}_{r}\right) d S$.

One can then show that an increment of dissipated energy is equal to

$$
\begin{aligned}
d \Phi=\frac{1}{2} \int_{\Gamma}\left(\underline{F} d \underline{u}-\underline{u} d \underline{F}+\underline{F} d \underline{u}_{r}+\underline{u}_{r} d \underline{F}\right) d S & \\
& -d \Psi_{r}
\end{aligned}
$$

The details of the calculations leading to these expressions of $\Psi$ and $d \Phi$ are presented in the annex. For the fictitious transformation, $d \Psi_{r}$ and $d \underline{u}_{r}$ are zero because the unloaded state remains unchanged. Thus, $d \Phi^{f}$ is equal to

$d \Phi^{f}=\frac{1}{2} \int_{\Gamma}\left(\underline{F} d \underline{u}-\underline{u} d \underline{F}+\underline{u}_{r} d \underline{F}\right) d S$.

From Equations 36, 52, 58 and 59, the increment of plastic dissipated energy is equal to

$d \Phi^{p}=\int_{\Gamma} \underline{F} d \underline{u}_{r} d S-2 d \Psi_{r}$

\subsection{Conservation of the terms of the energy balance}

Using 56 , the energy balance 54 can be written in the following form:

$\mathcal{E}=\Psi_{a}+\Psi_{r}-W_{e x t}+\Phi$.

We assume that the model substitution criterion is verified. Therefore, at any time, the plastic, elastic and fictitious energies of the two models are equal. The total energies, which do not vary with time and are defined to within a constant, can be considered to be equal for the two models. Finally, we assume that the free residual energies of the two models are equal. This is a strong, but necessary assumption in order to get to the desired result. Thus, the following equality of the energies of the equivalent model, denoted $(\stackrel{\wedge}{)}$, and the energies of the reference model, denoted (. ), holds:

$\left(\hat{\mathcal{E}}, \hat{\Phi}^{f}, \hat{\Phi}^{p}, \hat{\Psi}_{r}\right)=\left(\breve{\mathcal{E}}, \breve{\Phi}^{f}, \breve{\Phi}^{p}, \breve{\Psi}_{r}\right), \quad \forall t$.

In order to show that the other terms of the energy balance are also preserved, one proceeds by induction between a time $t$ and the immediately subsequent time $t+d t$. The recurrence relation is assumed to be verified at time $t$ :

$\left(\hat{\Psi}_{a}^{t}, \hat{W}_{e x t}^{t}\right)=\left(\breve{\Psi}_{a}^{t}, \breve{W}_{e x t}^{t}\right)$

From Assumption 1, the boundary conditions are applied as prescribed loads at the boundary of the structure, and from Assumption 2 the applied load $\underline{F}$ can be defined using a reference loading $\underline{F}_{0}$ and a loading coefficient $\lambda$. Since both models are subjected to the same boundary conditions,

$\underline{\hat{F}}=\underline{\breve{F}}=\underline{F}=\lambda \underline{F}_{0}, \quad$ over $\Gamma$,

and the loading increment verifies

$d \underline{\hat{F}}=d \underline{\breve{F}}=d \underline{F}=d \lambda \underline{F}_{0}, \quad$ over $\Gamma$.

The model substitution criterion being verified, the increments of plastic and fictitious dissipated energy are the same for both models. Therefore, Equations 59 and 60 lead to

$$
\begin{aligned}
\int_{\Gamma}\left(\underline{F}^{t} d \underline{\hat{u}}-\left(\underline{\hat{u}}^{t}-\underline{\hat{u}}_{r}^{t}\right) d \underline{F}\right) d S= \\
\int_{\Gamma}\left(\underline{F}^{t} d \underline{\breve{u}}-\left(\underline{u}^{t}-\underline{\breve{u}}_{r}^{t}\right) d \underline{F}\right) d S,
\end{aligned}
$$

$\int_{\Gamma}\left(\underline{F}^{t} d \underline{\hat{u}}_{r}\right) d S=\int_{\Gamma}\left(\underline{F}^{t} d \underline{\breve{u}}_{r}\right) d S$.

Using 64 and 65 :

$$
\begin{aligned}
\int_{\Gamma} \underline{F}_{0}\left(\lambda^{t} d \underline{\hat{u}}-\left(\underline{\hat{u}}^{t}-\underline{\hat{u}}_{r}^{t}\right) d \lambda\right) d S= \\
\int_{\Gamma} \underline{F}_{0}\left(\lambda^{t} d \underline{\breve{u}}-\left(\underline{\breve{u}}^{t}-\underline{\breve{u}}_{r}^{t}\right) d \lambda\right) d S,
\end{aligned}
$$


$\int_{\Gamma} \underline{F}_{0}\left(\lambda^{t} d \underline{\hat{u}}_{r}\right) d S=\int_{\Gamma} \underline{F}_{0}\left(\lambda^{t} d \underline{\breve{u}}_{r}\right) d S$

The recurrence relation at time $t$ yields $\hat{\Psi}_{a}^{t}=\breve{\Psi}_{a}^{t}$. Therefore,

$\int_{\Gamma} \lambda^{t} \underline{F}_{0}\left(\underline{\hat{\underline{u}}}^{t}-\underline{\hat{\hat{u}}}_{r}^{t}\right) d S=\int_{\Gamma} \lambda^{t} \underline{F}_{0}\left(\underline{\breve{u}}^{t}-\underline{\breve{u}}_{r}^{t}\right) d S$.

Using Equation 70, the system consisting of Equations 68 and 69 enables one to write

$$
\begin{aligned}
\int_{\Gamma} \underline{F}_{0} d \underline{\hat{u}} d S & =\int_{\Gamma} \underline{F}_{0} d \underline{u} d S, \\
\int_{\Gamma} \underline{F}_{0} d \underline{\hat{u}}_{r} d S & =\int_{\Gamma} \underline{F}_{0} d \underline{\breve{u}}_{r} d S .
\end{aligned}
$$

Indeed, we have

$\int_{\Gamma} \underline{F}_{0}\left(d \underline{\hat{u}}-d \underline{\hat{\hat{u}}}_{r}\right) d S=\int_{\Gamma} \underline{F}_{0}\left(d \underline{\breve{u}}-d \underline{\breve{u}}_{r}\right) d S$,

which, using 70 , leads to

$$
\begin{aligned}
\int_{\Gamma} \underline{F}^{t+d t}\left(\underline{\hat{u}}^{t+d t}-\underline{\hat{u}}_{r}^{t+d t}\right) d S= \\
\int_{\Gamma} \underline{F}^{t+d t}\left(\underline{\breve{u}}^{t+d t}-\underline{\breve{u}}_{r}^{t+d t}\right) d S .
\end{aligned}
$$

Thus, indeed, $\Psi_{a}$ is preserved when switching models at time $t+d t$ :

$\hat{\Psi}_{a}^{t+d t}=\breve{\Psi}_{a}^{t+d t}$.

The use of Expression 61 of the energy balance enables one to establish the recurrence relation at $t+d t$ :

$\left(\hat{\Psi}_{a}^{t+d t}, \hat{W}_{e x t}^{t+d t}\right)=\left(\breve{\Psi}_{a}^{t+d t}, \breve{W}_{e x t}^{t+d t}\right)$.

The initialization of the recurrence relation is verified because the two models are identical at the beginning of the loading and remain so as long as one remains within the loading surface of the material.

\section{Numerical implementation for a one- dimensional problem}

The model substitution method can be used in order to obtain the cohesive law analytically if the cohesive model is simple enough (Cazes et al., 2009). In the present paper, we used the finite element method for the analysis of a one-dimensional beam of length $L=100 \mathrm{~mm}$ and crosssectional area $S=10 \mathrm{~mm}^{2}$ solicited in traction. The expression of the plasticity threshold function used, which is the same for the two models, is

$f(\tilde{\sigma}, p)=|\tilde{\sigma}|-R(p)$

with

$R(p)=E\left(\varepsilon_{0}+\frac{k}{1-k} p\right)$, where $k$ and $\varepsilon_{0}$ are parameters of the material. The calculation of the damage variable was carried out according to the decreasing exponential law:

$$
\begin{array}{lll}
D=0, & \text { if } \kappa \leq \kappa_{0}, \\
D=e^{-A\left(\kappa-\kappa_{0}\right)}, & & \text { if } \kappa>\kappa_{0} .
\end{array}
$$

where $A$ and $\kappa_{0}$ are material constants. The variable $z$ was chosen to be equal to the accumulated plastic strain $(z=p)$. For the reference model, $\kappa$ is obtained from Relations 17 and 18, whereas for the equivalent model Equation 24 suffices. The calculations were performed using the following material parameters: $E=200000 \mathrm{MPa}, \varepsilon_{0}=0.02$, $k=0.2, A=50, \kappa_{0}=0.04$ and $c=16 \mathrm{~mm}^{2}$. Figure 5 shows the material's traction curve for a monotonically increasing loading.

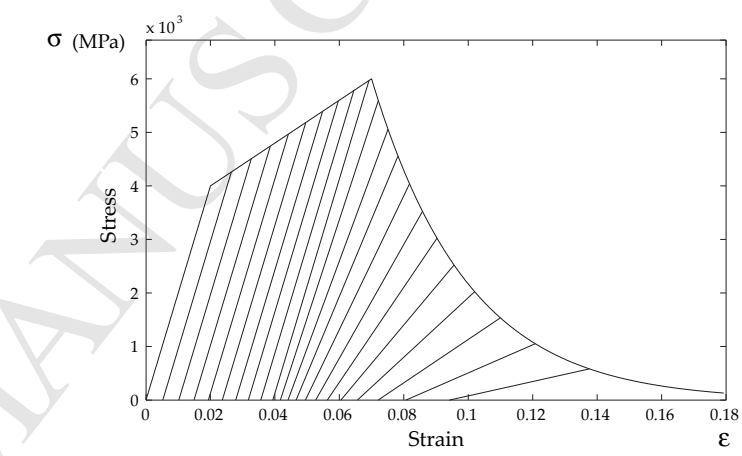

Figure 5: The traction curve of the continuous material

Three stages can be identified on this curve:

1) the elastic stage,

2) the hardening plastic stage,

3) and the softening plastic-damageable stage.

\subsection{The reference problem}

In order to set the position of the localized damage zone, a reduced-section zone of length $L_{0}=20 \mathrm{~mm}$ was defined at the center of the beam. The cross section of this beam segment was determined by the coefficient $\alpha=0.99$ such that the section is equal to $(1-\alpha) S$. The beam was fixed at the left end $\left(u_{0}=0\right)$ and subjected to a load $F$ at the other end. The geometry of the beam is shown in Figure 6.

a)

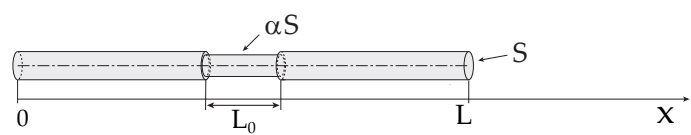

b)

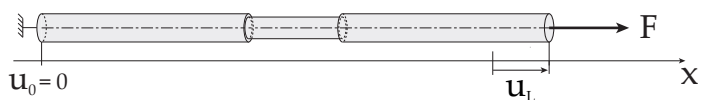

Figure 6: Geometry and loading (a) before deformation and (b) after deformation 


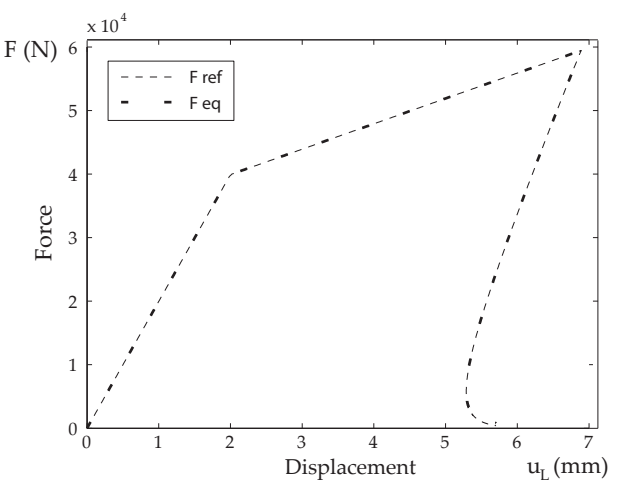

Figure 7: The load-displacement curve

The problem was discretized through the finite element method into 81 quadratic elements. The tangent matrices of the problem were calculated according to de Borst et al. (1999). The resolution required the use of a control scheme because of the snap-back phenomenon which can be observed on the load-displacement curve of Figure 7 . This control can be achieved with a method specific to dissipative problems: see, e.g., Gutiérrez (2004); Lorentz and Badel (2004). For this one-dimensional example, it was obtained by enforcing the strain of the central element of the beam. The numerical increments of plastic and fictitious dissipated energy, respectively $\Delta \breve{\Phi}^{p}$ and $\Delta \breve{\Phi}^{f}$, were saved in order to be reused during the resolution of the equivalent problem.

Figures 8, 9, and 10 show the evolutions obtained over the length of the beam for the regularized strain $\bar{z}$, the accumulated plastic strain $p$ and the damage $D$.

$\overline{\mathrm{Z}}$

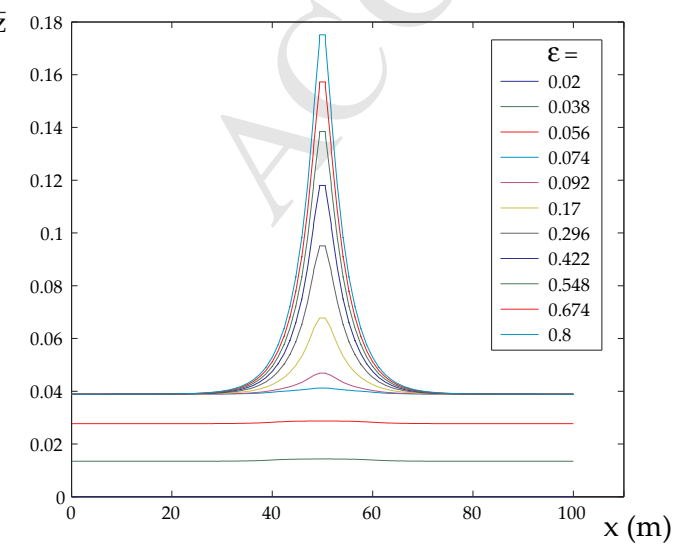

Figure 8: Regularized strain $\bar{z}$

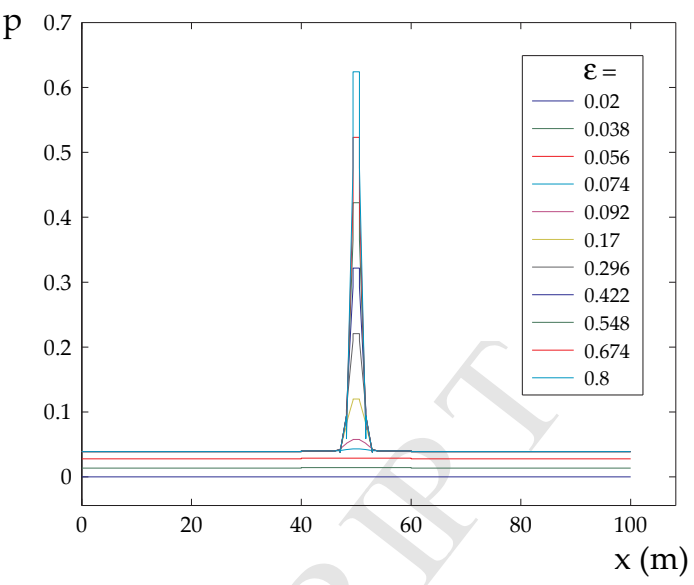

Figure 9: Accumulated plastic strain $p$

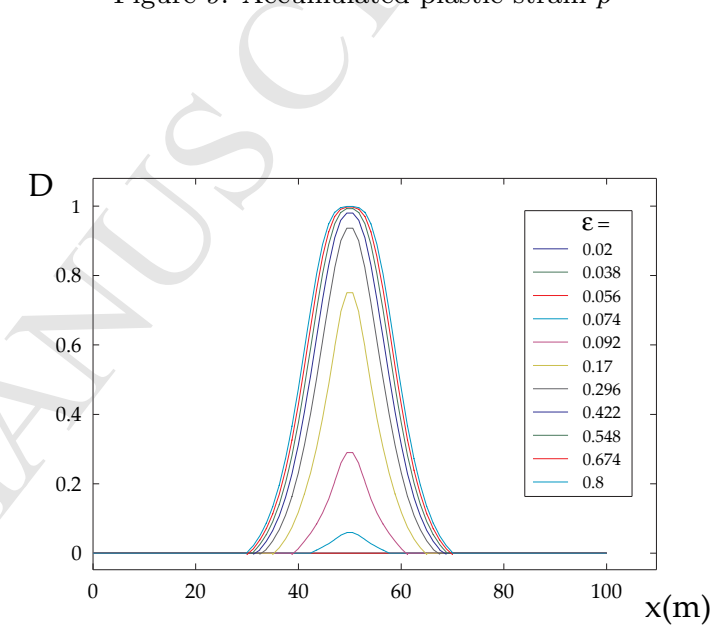

Figure 10: Damage $D$

\subsection{The equivalent problem}

For the equivalent problem, the beam was not given a reduced cross section in order for each point of the volume to be facing a point of the discontinuity and for the cohesive law obtained to be independent of the coefficient $\alpha$ used for the resolution of the reference problem. An equivalent cross-section $\hat{S}$ was calculated such that the strain energy would be the same for both models in the initial linear elastic stage. The resolution of the equivalent problem required a specific implementation because the cohesive law was built incrementally from the model substitution criterion defined by Equation 53. The only information being carried from the reference model to the equivalent model consisted of the increments of dissipated energy $\Delta \breve{\Phi}^{p}$ and $\Delta \breve{\Phi}^{f}$ and the load steps from the resolution of the reference problem. The discontinuity was assumed to occur in the middle of the beam, at the point of abscissa $x_{0}=\frac{L}{2}$. The beam geometry of the equivalent problem is shown in Figure 11. 


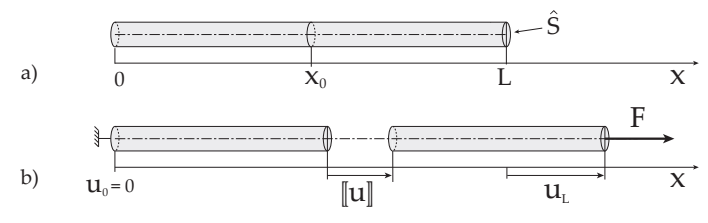

Figure 11: Geometry of the beam and loading (a) before deformation and (b) after deformation

Let $G$ be the set such that $G=\left[0, x_{0}[\cup] x_{0}, L\right]$ and let us consider the space $\mathcal{C}$ of the continuous, kinematically admissible scalar fields over $G$. The resolution is based on the following weak formulation of the problem :

$\mathcal{A}\left(d u^{*}\right)+\mathcal{B}\left(d \sigma^{s *}\right)=0, \quad \forall\left(d u^{*}, d \sigma^{s *}\right) \in(\mathcal{C}, \mathbb{R})$,

where $\mathcal{A}$ and $\mathcal{B}$ verify

$$
\begin{aligned}
\mathcal{A}\left(d u^{*}\right) & =-S \int_{G} d \varepsilon C d \varepsilon^{*} d x-S d \sigma^{s} d \llbracket u \rrbracket^{*}+d F d u_{L}^{*}, \\
\mathcal{B}\left(d \sigma^{*}\right) & =2 \frac{d \sigma^{s *}}{\sigma^{s}}\left(I_{l o c} d \breve{\Phi}^{f}-d \hat{\Phi}^{f}\right),
\end{aligned}
$$

with

$$
\varepsilon=\frac{\partial u}{\partial x}
$$

$\llbracket u \rrbracket=u^{+}\left(x_{0}\right)-u^{-}\left(x_{0}\right)$,

$u_{L}=u(L)$,

and $C$ the tangent operator :

$d \sigma=C d \varepsilon$

According to 53, the model substitution criterion requires that

$d \hat{\Phi}_{s}^{f}=I_{l o c} d \breve{\Phi}^{f}$

and, according to 50,

$d \hat{\Phi}_{s}^{f}=\frac{S}{2}\left(\sigma^{s} d \llbracket u \rrbracket-\left(\llbracket u \rrbracket-\llbracket u \rrbracket^{p}\right) d \sigma^{s}\right)$.

If Variables $m$ and $d v$ are defined by

$m=S \frac{\llbracket u \rrbracket-\llbracket u \rrbracket^{p}}{\sigma^{s}}$,

$d v=\frac{I_{l o c} d \breve{\Phi}^{f}}{\sigma^{s}}$

83 can be rewritten as

$\mathcal{B}\left(d \sigma^{s^{*}}\right)=d \sigma^{s *}\left(2 d v-S d \llbracket u \rrbracket+m d \sigma^{s}\right)$.

Operators $\mathrm{A}_{\mathrm{L}}, \mathrm{B}$ and $\mathrm{T}$, which connect the kinematic variables to the vector of the nodal displacements $\mathrm{U}$, are defined by

$$
u_{L}=\mathrm{A}_{\mathrm{L}} \mathrm{U}, \quad \varepsilon=\mathrm{BU}, \quad \llbracket u \rrbracket=\mathrm{TU} .
$$

The discretization of Equations 82 and 92 from these operators leads to the following system:

$$
\left(\begin{array}{cc}
\mathrm{K} & S \mathrm{~T}^{\mathrm{T}} \\
S \mathrm{~T} & -m
\end{array}\right)\left(\begin{array}{c}
\mathrm{dU} \\
\mathrm{d} \sigma^{\mathrm{s}}
\end{array}\right)=\left(\begin{array}{c}
\mathrm{A}_{\mathrm{L}}^{\mathrm{T}} d F \\
2 d v
\end{array}\right)
$$

with

$\mathrm{K}=S \int_{G} \mathrm{~B}^{\mathrm{T}} C \mathrm{~B} d x$

In order for the scale change criterion 53 to be verified, the conservation of the dissipated plastic energy must also be enforced:

$d \hat{\Phi}_{s}^{p}=I_{l o c} d \breve{\Phi}^{p}$.

Equations 32 lead to the following expression of $d \llbracket u \rrbracket^{p}$ :

$d \llbracket u \rrbracket^{p}=\frac{d \breve{\Phi}^{p}}{S \sigma^{s}}$

Then, System 94 is discretized in time and solved using a Newton-Raphson algorithm. The value of $\llbracket u \rrbracket^{p}$ is calculated from Equation 97, discretized in time according to an explicit Euler scheme. The value of $\llbracket u \rrbracket^{p}$ obtained is necessary in order to calculate $m$ in the next time step (Equation 91).

This resolution enabled us to determine the cohesive law represented in Figure 12. Figure 13 represents the energies dissipated by the two models. The good correspondence between the two curves shows that the scale change criterion was indeed verified. Figure 14 compares the free energies of the two models as functions of time. This time, the good correspondence between the two curves confirms that the other terms of the energy balance were preserved when switching models (5.2).

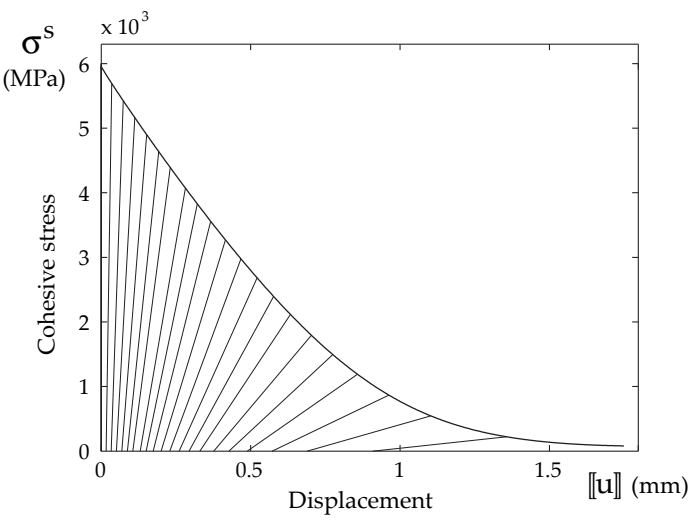

Figure 12: Traction curve of the cohesive model 


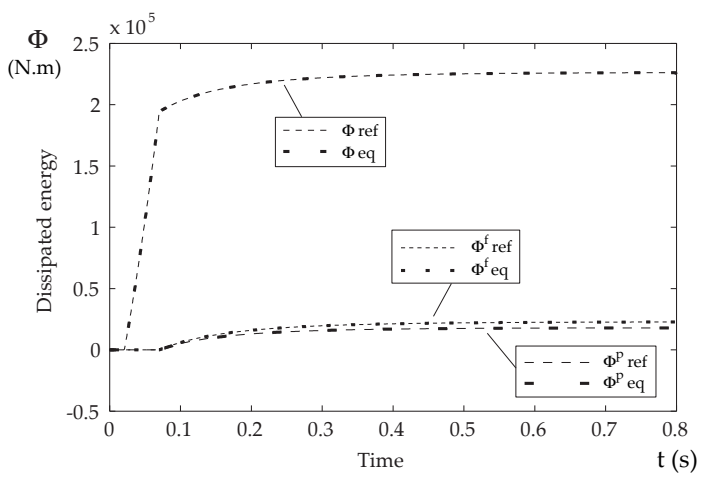

Figure 13: Dissipated energies of the two models

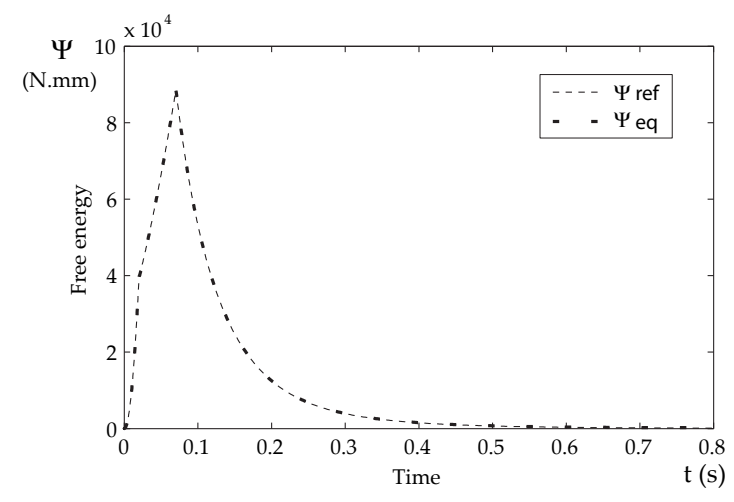

Figure 14: Free energies of the two models

\section{Discussion}

In this section, we take a new look at the assumptions made during the construction of the method and discuss in detail the conditions of its extension to multidimensional test cases.

One of the first assumptions made is that no energy is stored within the microstructure of the material. Thus, one gets a simplified expression of the increment of dissipated plastic energy, which reduces to an increment of plastic work (Equations 22 and 32). In fact, one often introduces a coefficient which represents the proportion of the plastic work dissipated as heat during the fracture. If one were to use the method taking into account thermal effects, this coefficient should be used to calculate the increase in the material's temperature correctly. A temperature jump across the discontinuity could also be allowed, as in Fagerström and Larsson (2008).

A second important assumption made is that the residual free energies of the two models must be equal in order for the terms of the energy balance to be preserved when switching models. This energy is due to the presence of residual stresses in the material after unloading. Even though this is a strong assumption, it was perfectly verified in the one-dimensional test case we studied because the residual stress field was zero over the entire length of the beam and the residual free energy was also zero.

For the reference model chosen, the effect of the regularization begins only with the occurrence of damage, which also corresponds to the beginning of the softening part of the material's behavior. The use of this type of model is to be preferred for the calculation of the cohesive law because the reference model and the equivalent model are identical throughout the hardening part of the material's behavior. This is justified both physically, because there is no reason to regularize the solution as long as there is no risk of localization, and numerically, because the tangent matrices remain symmetrical for as long as hardening behavior lasts.

The initial stiffness of the cohesive model obtained through the model substitution remains infinite until the threshold stress is reached. Therefore, this model belongs to the family of extrinsic models as opposed to intrinsic models which have finite initial stiffness. This result is consistent because during the whole hardening phase no dissipated energy is transferred to the cohesive zone and the multiplier $\mu$ achieves the contact between the corresponding nodes on both sides of the discontinuity. The extrinsic character of the law can lead to numerical implementation problems because of some terms of the stiffness matrix which should be infinite prior to the initiation of the cohesive zone. This problem vanishes with methods in which the cohesive law is embedded in an element, and can be worked around using mixed interface elements (Lorentz, 2008) or a truly-mixed formulation (Bruggi and Venini, 2007). Some care must be taken when using these formulations because the discretisation must satisfy the inf-sup condition for the calculation to be stable. The numerical implementation of intrinsic laws can also present difficulties because of oscillations of the stress field, depending on the numerical integration scheme being used (J.C.J. Schellekens, 1993).

If the localized damage indicator $I_{l o c}$ is not used and the whole dissipated energy of the reference model is transferred to the cohesive model, the resulting cohesive law contains an increasing part before it starts decreasing. This type of law is not advisable for usual applications of cohesive zones because it creates a diffuse cracking zone around the main crack: see, e.g., Planas et al. (2003)).

This method can be generalized to multidimensional problems in mode I by replacing the global scale change criterion with a local criterion to be verified at each point of the discontinuity. The dissipated surface energy to be introduced into the cohesive model is calculated by integrating the dissipated volume energy over a segment which is perpendicular to the discontinuity surface at the point being considered. This leads to the following criterion, 
which is illustrated by Figures 15 and 16:

$\hat{\phi}_{s}^{p}=\int_{-l}^{l}\left(\breve{\phi}^{p}-\hat{\phi}^{p}\right) d l$,

$\hat{\phi}_{s}^{e}=\int_{-l}^{l}\left(\breve{\phi}^{e}-\hat{\phi}^{e}\right) d l$

where the length $2 l$ of the integration segment is determined so that it runs across the localized damage zone surrounding the crack. If hardening behavior exists in the zone surrounding the discontinuity, it is assumed that $d \breve{\phi}^{p}=d \hat{\phi}^{p}$ and $d \breve{\phi}^{e}=d \hat{\phi}^{e}$. In the case of softening behavior, the zone surrounding the discontinuity is unloaded because of the opening of the crack, and $d \hat{\phi}^{p}=d \hat{\phi}^{e}=0$. This enables one to introduce a local localization indicator $i_{\text {loc }}$ defined at each point of the discontinuity. Then, the model substitution criterion becomes

$\begin{aligned} d \hat{\phi}_{s}^{p} & =i_{l o c} \int_{-l}^{l} d \breve{\phi}^{p} d l, \\ d \hat{\phi}_{s}^{e} & =i_{l o c} \int_{-l}^{l} d \breve{\phi}^{e} d l .\end{aligned}$

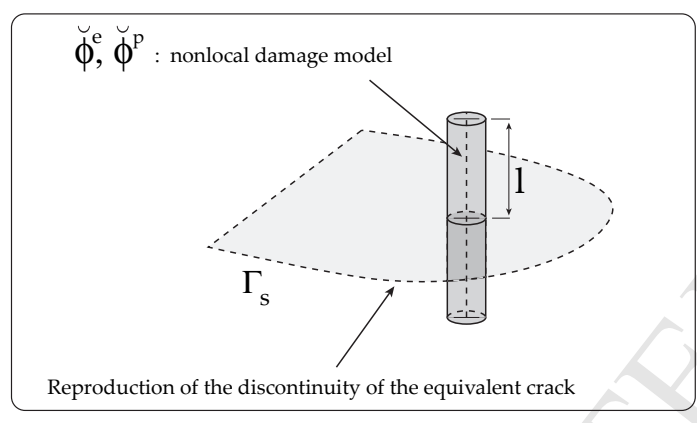

Figure 15: Dissipated energies of the reference model

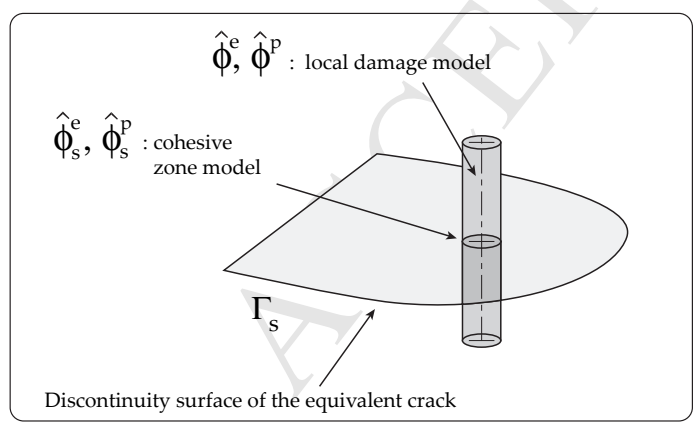

Figure 16: Dissipated energies of the equivalent model

\section{Conclusion}

The vast number of works which combine continuous models and discontinuous models is an indication of the complementarity of the two approaches. These works can be categorized into two groups:
1) the works which tend to use a continuous model and a discontinuous model side-by-side to treat the two phases of the fracture separately and, thus, achieve a more refined modeling of the material's behavior or a more robust numerical implementation,

2) the works which attempt to superimpose a discontinuous model and a continuous model in order to get two equivalent and interchangeable models.

The works reported in the present paper belong to both Group 1, because the model obtained combines a 'local' continuous model and a cohesive model, and Group 2, because the cohesive law is built from the regularized reference model. The method, which had been presented in a previous paper for damage models, was extended in the present paper to materials which can experience plastic strains. This required

- the introduction of a plastic displacement jump which must be taken into account in the thermodynamic description of the cohesive zone,

- the decomposition of the dissipated energy into a plastic part and an elastic part, along with the development of a model substitution criterion which enforces the preservation of these two energies during the construction of the cohesive law,

and a specific numerical implementation of the equivalent problem enabling the solution to be calculated from the model substitution criterion with no a priori knowledge of the form of the cohesive law.

The method can be used for the determination of the parameters of a model combining the continuous and discontinuous descriptions of the material. This would enable one to avoid having to solve an inverse problem for a material whose continuous, regularized constitutive behavior is already known. This constitutive behavior can be identified more easily because, then, the model contains only a continuous description and the resolution of the inverse problem can be based on local quantities, such as the width of the damage zone. This method, once implemented for multidimensional models, could also help one understand some poorly known aspects of cohesive models (described in detail in, e.g., Bažant (2002)). In particular, it could be interesting to study the relation between the traction behavior and the shear behavior of the cohesive zone, or the influence of triaxiality on the form of the cohesive law. This evolution seems to be possible provided that the global energy criterion is replaced by a local criterion defined at each point of the discontinuity.

\section{Acknowledgements}

This work was supported by the SAFRAN group under Contract n ${ }^{\circ}$ YHMMV_2007_012. 


\section{References}

Aifantis, E., 1984. On the structural origin of certain inelastic models. Journal of Engineering Materials and Technology 106, 326330 .

Areias, P., Belytschko, T., 2005. Analysis of three-dimensional crack initiation and propagation using the extended finite element method. International Journal for Numerical Methods in Engineering 63 (5), 760-788

Barenblatt, G., 1962. The mathematical theory of equilibrium cracks in brittle fracture. Advances in Applied Mechanics 7, 55-129.

Bažant, Z., 2002. Concrete fracture models: testing and practice. Engineering Fracture Mechanics 69 (2), 165-205.

Bažant, Z., Belytschko, T., 1985. Wave propagation in a strainsoftening bar: exact solution. Journal of Engineering Mechanics 111 (3), 381-389.

Bažant, Z., Oh, B., 1983. Crack band theory for fracture of concrete. Materials and Structures 16 (3), 155-177.

Belytschko, T., Black, T., 1999. Elastic crack growth in finite elements with minimal remeshing. International Journal for Numerical Methods in Engineering 45 (5), 601-620.

Benvenuti, E., Tralli, A., Ventura, G., 2008. A regularized xfem model for the transition from continuous to discontinuous displacements. International Journal for Numerical Methods in Engineering 74 (6), 911-944.

Bruggi, M., Venini, P., 2007. A Truly Mixed Approach for CohesiveCrack Propagation in Functionally Graded Materials. Mechanics of Advanced Materials and Structures 14 (8), 643-654.

Cazes, F., Coret, M., Combescure, A., Gravouil, A., 2009. A thermodynamic method for the construction of a cohesive law from a nonlocal damage model. International Journal of Solids and Structures 46 (6), 1476-1490.

Comi, C., Mariani, S., Perego, U., 2002. On the transition from continuum nonlocal damage to quasi-brittle discrete crack models. In: GIMC_2002 Third Joint Conference of Italian Group of Computational Mechanics and Ibero-Latin American Association of Computational Methods in Engineering.

Comi, C., Mariani, S., Perego, U., 2007. An extended FE strategy for transition from continuum damage to mode I cohesive crack propagation. International Journal for Numerical and Analytical Methods in Geomechanics 31 (2), 213-238.

Costanzo, F., Allen, D., 1995. A continuum thermodynamic analysis of cohesive zone models. International Journal of Engineering Science 33 (15), 2197-2219.

de Borst, R., Abellan, M., 2002. A numerical framework for continuum damage- discontinuum transition. Archives of Mechanics 54 (5-6), 377-387.

de Borst, R., Pamin, J., Geers, M., 1999. On coupled gradientdependent plasticity and damage theories with a view to localization analysis. European Journal of Mechanics/A Solids 18 (6), 939-962.

Dufour, F., Pijaudier-Cabot, G., Choinska, M., Huerta, A., 2008. Extraction of a crack opening from a continuous approach using regularized damage models. Computers \& Concrete 5 (4), 375388 .

Dugdale, D., 1960. Yielding of steel sheets containing slits. Journal of the Mechanics and Physics of Solids 8 (2), 100-108.

Fagerström, M., Larsson, R., 2008. A thermo-mechanical cohesive zone formulation for ductile fracture. Journal of the Mechanics and Physics of Solids 56, 3037-3058.

Griffith, A., 1920. The phenomena of rupture and flow in solids. Philosophical Transactions of the Royal Society CCXXI (A), 163198.

Gurtin, M., 1979. Thermodynamics and the cohesive zone in fracture. Zeitschrift für Angewandte Mathematik und Physik (ZAMP) 30 (6), 991-1003.

Gutiérrez, M. A., 2004. Energy release control for numerical simulations of failure in quasi-brittle solids. Communications in Numerical Methods in Engineering 20 (1), 19-29.

Hillerborg, A., Modéer, M., Petersson, P., 1976. Analysis of crack formation and crack growth in concrete by means of fracture me- chanics and finite elements. Cement and Concrete Research 6 , $773-782$.

J.C.J. Schellekens, R. d. B., 1993. On the numerical integration of interface elements. International Journal for Numerical Methods in Engineering 36 (1), 43-66.

Ladevèze, P., 1992. A damage computational method for composite structures. Computers and Structures 44 (1-2), 79-87.

Legrain, G., Dufour, F., Huerta, A., Pijaudier-Cabot, G., 2007. Extraction of crack opening from a nonlocal damage field. In: IX International Conference on Computational Plasticity (COMPLAS), Fundamentals and Applications. Vol. 1. pp. 462-465.

Lemaitre, J., Chaboche, J.-L., 1988. Mécanique des matériaux solides. Dunod.

Lorentz, E., 2008. A mixed interface finite element for cohesive zone models. Computer Methods in Applied Mechanics and Engineering 198 (2), 302-317.

Lorentz, E., Badel, P., 2004. A new path-following constraint for strain-softening finite element simulations. International Journal for Numerical Methods in Engineering 60 (2), 499-526.

Mariani, S., Perego, U., 2003. Extended finite element method for quasi-brittle fracture. International Journal for Numerical Methods in Engineering 58 (1), 103-126.

Mazars, J., 1984. Application de la mécanique de l'endommagement au comportement non-linéaire et à la rupture du béton de structure. Ph.D. thesis, ENSET.

Mazars, J., Pijaudier-Cabot, G., 1996. From damage to fracture mechanics and conversely : a combined approach. International Journal of Solids and Structures 33 (20), 3327-3342.

Mediavilla, J., Peerlings, R., Geers, M., 2006. An integrated continuous-discontinuous approach towards damage engineering in sheet metal forming processes. Engineering Fracture Mechanics 73 (7), 895-916.

Moës, N., Belytschko, T., 2002. Extended finite element method for cohesive crack growth. Engineering Fracture Mechanics 69 (7), 813-833.

Moës, N., Dolbow, J., Belytschko, T., 1999. A finite element method for crack growth without remeshing. International Journal for $\mathrm{Nu}$ merical Methods in Engineering 46 (1), 131-150.

Oliver, J., 1996. Modelling strong discontinuities in solid mechanics via strain softening constitutive equations. part 1: Fundamentals. International Journal for Numerical Methods in Engineering 39 (21), 3575-3600.

Oliver, J., Huespe, A., Pulido, M., Chaves, E., 2002. From continuum mechanics to fracture mechanics : the strong discontinuity approach. Engineering Fracture Mechanics 69 (2), 113-136.

Patzák, B., Jirásek, M., 2004. Adaptive resolution of localized damage in quasi-brittle materials. Journal of Engineering Mechanics 130 (6), 720-732.

Peerlings, R., Geers, M., de Borst, R., Brekelmans, W., 2001. A critical comparison of nonlocal and gradient-enhanced softening continua. International Journal of solids and Structures 38 (4445), 7723-7746.

Pijaudier-Cabot, G., Bažant, Z., October 1987. Nonlocal damage theory. Journal of Engineering Mechanics 113 (10), 1512-1533.

Planas, J., Elices, M., F.J., G. G., D.A., G., Arbilla, C. I., 2003. Generalizations and specializations of cohesive crack models. Engineering Fracture Mechanics 70 (14), 1759-1776.

Planas, J., Elices, M., Guinea, G., 1993. Cohesive cracks versus nonlocal models : Closing the gap. International Journal of Fracture 63 (2), 173-187.

Rodríguez-Ferran, A., Huerta, A., 2000. Error estimation and adaptivity for nonlocal damage models. International Journal of Solids and Structures 37 (48-50), 7501-7528.

Simo, J., Oliver, J., Armero, F., 1993. An analysis of strong discontinuities induced by strain-softening in rate-independent inelastic solids. Computational Mechanics 12 (5), 277-296.

Simone, A., Wells, G., Sluys, L., 2003. From continuous to discontinuous failure in a gradient-enhanced continuum damage model. Computer Methods in Applied Mechanics and Engineering 192 (41), 4581-4607.

Wells, G., Sluys, L., 2001. A new method for modelling cohesive 
cracks using finite elements. International Journal for Numerical Methods in Engineering 50 (12), 2667-2682.

\section{A. Annex - Calculation of the free energy and dis- sipated energy}

This annex describes how expressions of the free energy and of an increment of dissipated energy for the structure can be derived from data defined at the boundary of Domain $\Gamma$. We are considering the most general case in which the damageable elastic-plastic domain contains a discontinuity $\Gamma_{s}$. The continuous part of the model is elastic-plastic damageable, and the discontinuity is described through a cohesive model similar to that of 3.2. The solution of the problem is assumed to be known at time $t$, and the fields associated with this solution are denoted $(.)_{t}$. At time $t$, the threshold functions verify:

$$
\begin{aligned}
f\left(\underline{\underline{\sigma}}_{t}, p_{t}\right) & \leq 0, & & \text { over } \Omega, \\
f_{s}\left(\underline{\sigma}_{t}^{s}, v_{t}^{k}\right) & \leq 0, & & \text { over } \Gamma_{s} .
\end{aligned}
$$

If, starting from this solution, the structure is completely unloaded until a free boundary is achieved over $\Gamma$, one obtains the residual state. The fields associated with this state are denoted $(.)_{r}$. The transformation which leads from the known state at $t$ to the residual state is described through the evolution of a loading factor $\lambda$ which varies from 0 to 1 so that:

$\underline{F}(\lambda)=(1-\lambda) \underline{F}_{t}, \quad$ over $\Gamma$.

During this transformation, the variables are expressed as functions of the parameter $\lambda$. According to Assumption 3, the unloaded state is included in the elastic domain of the material prior to the unloading: thus,

$$
\begin{aligned}
f\left(\underline{\underline{\sigma}}_{r}, p_{t}\right) & \leq 0, & & \text { over } \Omega \\
f_{s}\left(\underline{\sigma}_{r}^{s}, v_{t}^{k}\right) & \leq 0 . & & \text { over } \Gamma_{s} .
\end{aligned}
$$

The following displacement fields and displacement jumps are proposed as the solutions of the unloading problem:

$$
\begin{aligned}
\underline{u}(\lambda) & =(1-\lambda) \underline{u}_{t}+\lambda \underline{u}_{r}, \\
\llbracket \underline{u} \rrbracket(\lambda) & =(1-\lambda) \llbracket \underline{u} \rrbracket_{t}+\lambda \llbracket \underline{u} \rrbracket_{r} .
\end{aligned}
$$

The fields associated with plasticity and damage are assumed to remain constant during the transformation:

$$
\begin{aligned}
\underline{\underline{\varepsilon}}^{p}(\lambda) & =\underline{\underline{\varepsilon}}_{t}^{p}, \\
\llbracket \underline{u} \rrbracket^{p}(\lambda) & =\llbracket \underline{u} \rrbracket_{t}^{p}, \\
D(\lambda) & =D_{t} .
\end{aligned}
$$

In order to verify that the proposed solution is indeed the solution of the problem, one must test, for all values of $\lambda$ between 0 and 1 , the static admissibility equations, i.e.:

$$
\begin{aligned}
\underline{\operatorname{div}}(\underline{\underline{\sigma}}(\lambda)) & =\underline{0}, & & \text { over } \Omega, \\
\underline{\underline{\sigma}}(\lambda) \underline{n} & =\underline{F}(\lambda), & & \text { over } \Gamma, \\
\underline{\sigma}^{s}(\lambda) & =\underline{\sigma}^{+}(\lambda) \underline{n}=\underline{\underline{\sigma}}^{-}(\lambda) \underline{n}, & & \text { over } \Gamma_{s} .
\end{aligned}
$$

From Equation 107, the strain field is equal to:

$$
\underline{\underline{\varepsilon}}(\lambda)=(1-\lambda) \underline{\underline{\varepsilon}}_{t}+\lambda \underline{\underline{\varepsilon}}_{r} .
$$

Since the plastic strain field is assumed to remain constant during the the transformation, one also has:

$\underline{\underline{\varepsilon}}^{e}(\lambda)=(1-\lambda) \underline{\underline{\varepsilon}}_{t}^{e}+\lambda \underline{\underline{\varepsilon}}_{r}^{e}$.

From Equation 111, the damage remains constant during the transformation. Therefore, the stresses are equal to:

$\underline{\underline{\sigma}}(\lambda)=(1-\lambda) \underline{\underline{\sigma}}_{t}+\lambda \underline{\underline{\sigma}}_{r}$

Equation 112 is verified for Fields $\underline{\sigma}_{t}$ and $\underline{\sigma}$, which are statically admissible. Therefore, Equation $1 \overline{\overline{1}} 2$ is also verified for $\underline{\underline{\sigma}}(\lambda)$. Expression 117 leads to the following expression of $\underline{\underline{\sigma}}(\lambda) \underline{n}$ over $\Gamma$ :

$\underline{\underline{\sigma}}(\lambda) \underline{n}=(1-\lambda) \underline{\sigma}_{t} \underline{n}+\lambda \underline{\sigma}_{r} \underline{n}$.

Besides, over $\Gamma$, one has:

$\underline{\underline{\sigma}}_{t} \underline{n}=\underline{F}_{t}$

$\underline{\underline{\sigma}} r \underline{n}=\underline{0}$.

Therefore:

$\underline{\underline{\sigma}}(\lambda) \underline{n}=(1-\lambda) \underline{F}_{t}=\underline{F}(\lambda) \quad$ over $\Gamma$,

which shows that Equation 113 is, indeed, verified for all values of $\lambda$. Equation 117 also shows that over $\Gamma_{s}$ one has:

$\underline{\underline{\sigma}}^{+}(\lambda) \underline{n}=(1-\lambda) \underline{\sigma}_{t}^{+} \underline{n}+\lambda \underline{\underline{\sigma}}_{r}^{+} \underline{n}$,

$\underline{\underline{\sigma}}^{-}(\lambda) \underline{n}=(1-\lambda) \underline{\sigma}_{t}^{-} \underline{n}+\lambda \underline{\underline{\sigma}}_{r}^{-} \underline{n}$.

Besides, from 108 and 110,

$\llbracket \underline{u} \rrbracket^{e}(\lambda)=(1-\lambda) \llbracket \underline{u} \rrbracket_{t}^{e}+\lambda \llbracket \underline{u} \rrbracket_{r}^{e}$.

Then, the linear behavior of the cohesive zone during the unloading leads to:

$\underline{\sigma}^{s}(\lambda)=(1-\lambda) \underline{\sigma}_{t}^{s}+\lambda \underline{\sigma}_{r}^{s}$.

Therefore, the equations of 114 are verified throughout the unloading. All we have left to do is verify that the transformation was indeed performed within the initial loading surface. The threshold functions $f$ and $f_{s}$ must be convex in order to ensure that the dissipated energy is positive. Thus:

$$
\begin{aligned}
f\left(\lambda \underline{\underline{\tilde{\sigma}}}_{r}+(1-\lambda) \underline{\underline{\tilde{\sigma}}}_{t},\right. & \left.p_{t}\right) \leq \\
& \lambda f\left(\underline{\underline{\tilde{\sigma}}}_{r}, p_{t}\right)+(1-\lambda) f\left(\underline{\underline{\tilde{\sigma}}}_{t}, p_{t}\right),
\end{aligned}
$$




$$
\begin{aligned}
f_{s}\left(\lambda \underline{\sigma}_{r}^{s}+(1-\lambda)\right. & \left.\underline{\sigma}_{t}^{s}, v_{t}^{k}\right) \leq \\
& \lambda f_{s}\left(\underline{\sigma}_{r}^{s}, v_{t}^{k}\right)+(1-\lambda) f_{s}\left(\underline{\sigma}_{t}^{s}, v_{t}^{k}\right) .
\end{aligned}
$$

Therefore, from Equations 102, 105, and 103, 106:

$$
\begin{aligned}
f\left(\underline{\underline{\tilde{\sigma}}}(\lambda), p_{t}\right) & \leq 0, & & \text { over } \Omega, \\
f_{s}\left(\underline{\sigma}^{s}(\lambda), v_{t}^{k}\right) & \leq 0, & & \text { over } \Gamma_{s},
\end{aligned}
$$

which shows that the transformation takes place inside the initial loading surface throughout the process, and Equations 109, 110, 111 are indeed verified.

The solution of the unloading problem can be used to get an expression of the free energy $\Psi$. Since the transformation is non-dissipative, the energy balance leads to:

$\Delta \Psi=\Delta \mathcal{W}_{\text {ext }}$.

Therefore, the variation of free energy can be calculated from the solution of the unloading problem:

$\Delta \Psi=\int_{\lambda=0}^{1} \underline{F}(\lambda) d \underline{u}$,

$\Delta \Psi=\int_{\lambda=0}^{1}(1-\lambda) \underline{F}_{t}\left(-\underline{u}_{t}+\underline{u}_{r}\right) d \lambda$,

$\Delta \Psi=-\frac{1}{2} \underline{F}_{t}\left(\underline{u}_{t}-\underline{u}_{r}\right)$.

This energy represents the difference between the residual free energy $\Psi_{r}$ and the free energy at time $t$, which we will denote simply $\Psi$. Thus:

$\Psi=\Psi_{a}+\Psi_{r}$,

with

$\Psi_{a}=\frac{1}{2} \int_{\Gamma} \underline{F}\left(\underline{u}-\underline{u}_{r}\right)$.

This justifies the use of Equations 56 and 57 in Section 5.1. For an arbitrary transformation, the energy balance leads to the following expression of the increment of dissipated energy:

$d \Phi=d \mathcal{W}_{e x t}-d \Psi$,

Therefore, using 134 and 135, one can recover Expression 58 of an increment of dissipated energy:

$d \Phi=\frac{1}{2} \int_{\Gamma}\left(\underline{F} d \underline{u}-\underline{u} d \underline{F}+\underline{F} d \underline{u}_{r}+\underline{u}_{r} d \underline{F}\right) d S$

$-d \Psi_{r}$. 